\title{
MeDe innovation fifth annual meeting and 3DMedical expo 2018
}

\author{
Sonia Mannan*,1 \\ ${ }^{1}$ Future Science Group, Unitec House, 2 Albert Place, London N31QB, UK \\ *Author for correspondence: s.mannan@futuremedicine.com
}

First draft submitted: 8 March 2018; Accepted for publication: 16 October 2018; Published online: 30 November 2018

This conference report summarizes highlights from the MeDe Innovation Fifth Annual Meeting and the 3DMedical Expo.

\section{MeDe innovation fifth annual meeting}

The MeDe Innovation Fifth Annual Conference took place on 20th January 2018 in Sheffield, UK [1]. This was an interesting event concerning medical device manufacture with a gathering of academics, industrialists, clinicians and regulatory body representatives.

The event was broken into three parts:

\section{- Session A: responsible innovation and the manufacture of new medical devices}

The event was kicked off with Suzanne Halliday from British Standards Institution (London, UK) discussing the regulatory perspectives that need to be considered when producing and reviewing medical devices. Tim Briggs from Royal National Orthopaedic Hospital (Stanmore, UK) showcased how much orthopedics have advanced over the last 50 years, discussing cases and projects he has worked on throughout his career. Finally, Edward Draper from JRI Orthopaedics (Sheffield, UK) presented an industrial argument before all three speakers formed a panel to answer questions raised by the audience.

\section{- Poster pitch and viewing}

Over $30 \mathrm{PhD}$ students were given the chance to pitch their posters for their projects in under $1 \mathrm{~min}$ before the start of the poster viewing session, giving us a quick summary of their research and a basis to ask further questions during the viewing session. Some of the areas presented included topics such as bioprinted skin for toxicity testing and 3D-manufactured maxillofacial implants.

\section{- Section B: the impact of MeDe innovation research}

The concluding part of the event consisted of talks on the types of research that have come about as a result of MeDe Innovation research. Steven Percival (5D Health Protection Group, Liverpool, UK) discussed the stratified design and manufacture of nonwoven collagen scaffolds, before David Grant (University of Nottingham, Nottingham, UK) described his teams work manufacturing fully bioresorbable multiphase fixation devices to order. These talks were followed by a presentation of Phil Coates (University of Bradford, Bradford, UK) and Paul Hatton's (University of Sheffield) work on minimally invasive implantation of bioactive materials, and the day was closed by Kenny Dalgarno discussing processes for in-clinic manufacture.

This conference provided valuable information about medical device regulations and the perspectives of individuals in the field of orthopaedics, as well as provide a insight into the projects young researchers are working on in the $3 \mathrm{D}$ printing and bioprinting world.

\section{DMedical expo}

The 3DMedical expo was held on 30th-31st January 2018 in Maastricht, The Netherlands [2]. The event was divided into several tracks covering the latest technological innovations in 3D printing in regard to medicine and health.

The event consisted of two conferences that were further broken down into dedicated sessions: 
3D medical printing event

- 3D bioprinting/3D biomaterials, 3D dental printing

- 3D medtech printing

- 3D medicine/pharmaceutics printing

\section{Health tech event}

There were a total of 35 speakers over the 2 days including representatives from SoftBank Robotics Europe (Paris, France), Boehringer Ingelheim (Ingelheim am Rhein, Germany), Materialise (Leuven, Belgium), Merck (NJ, USA), ACTA (Amsterdam, The Netherlands), Mobilab (Geel, Belgium), Uniklinik RWTH Aachen (Aachen, Germany), Maastricht University (Maastricht, The Netherlands), Tilburg University (Tilburg, The Netherlands), Eindhoven University of Technology (Eindhoven, The Netherlands), University of Pisa (Pisa, Italy), Katholieke Universiteit Leuven (Flanders, Belgium), TNO (The Hague, The Netherlands), Vektis (Utrecht, The Netherlands) and Tosoh SMD (OH, USA).

The 3D bioprinting and 3D dental printing sessions were held on the first day of the conference and included talks on the current status of having human tissue printed on demand, biofabrication of $3 \mathrm{D}$ in vitro models, hydrogels for tissue engineering, bioinks, 3D printing for dental implants and maxillofacial surgery, as well as some regulatory issues and ethical challenges that come with the advancement of these technologies.

The second and final day of the conference consisted of the 3D medtech printing and 3D pharma printing sessions. These tracks covered the use of $3 \mathrm{D}$ printing for surgical training, orthopedic implants and pharmaceutical manufacturing.

While at the 3DMedical Expo, interviews were obtained with a few of the speakers about their talks and key projects that they are working on. The interviews with Rik Jacobs (3D Systems Corporation, SC, USA) about 3D systems involvement in the dental industry; Boštjan Vihar (Institute IRNAS; Maribor, Slovenia) about open-source 3D bioprinting; Riley Reese (TNO) about how 3D printing can has transitioned from food to pharma and Laura Le Calvé (LCH Avocats; Paris, France) about the important legal issues concerning 3D-printed medical devices, will be published on the 3D printing and bioprinting community site 3DMedNet [3].

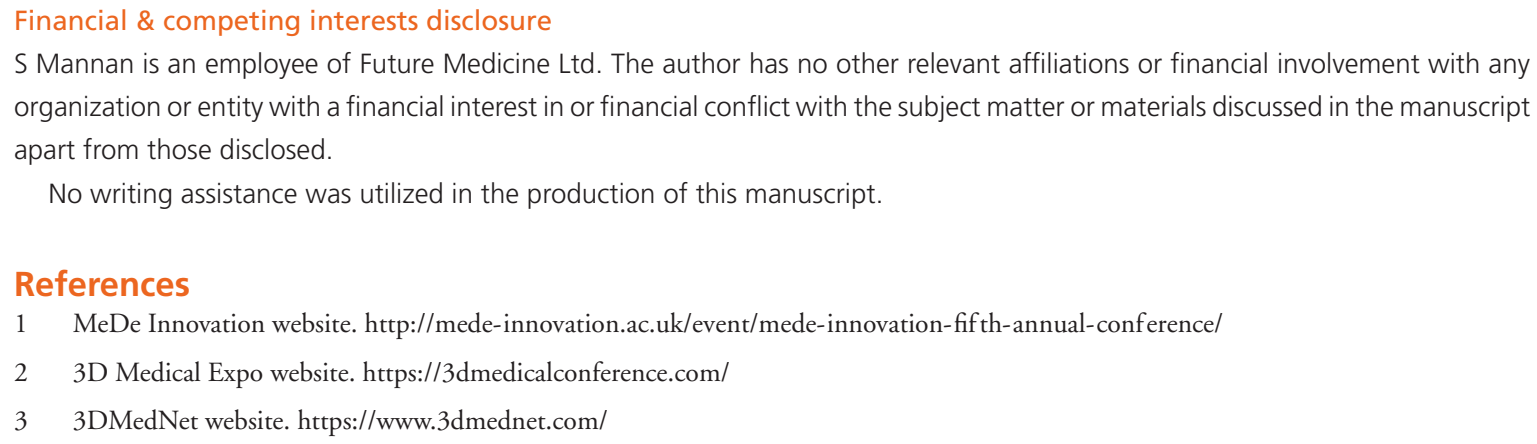

